\title{
Valoración del apoyo social durante el curso vital
}

\author{
Enrique Lombardo ${ }^{1}$ y Corina Soliverez ${ }^{2}$
}

\begin{abstract}
RESUMEN
El apoyo social es un constructo multidimensional que posee una perspectiva estructural y una perspectiva funcional. Desde la perspectiva estructural, se enfatiza la noción de red social, que corresponde a los vínculos que una persona posee y que percibe como significativas. La perspectiva funcional hace referencia a las funciones que desempeña esa red, los apoyos que proporciona y sus efectos en el bienestar del sujeto. Las redes se modifican a lo largo de la vida, en cuanto al tamaño y composición de sus vínculos, lo que puede repercutir en el apoyo que proporciona. Por este motivo, el objetivo de esta investigación fue explorar los apoyos más valorados y necesitados durante el curso vital. Para tal fin, se implementó un diseño no experimental transversal, descriptivo con combinación de metodologia cuantitativa y cualitativa. La muestra estuvo constituida por 120 sujetos, de ambos sexos, 30 por grupo de edad ( 20 a 30, 40 a 50, 60 a 70 y 80 a 90) residentes de la ciudad de Mar del Plata. Al total de la muestra se les administró: un cuestionario de datos sociodemográficos que permitió caracterizar la muestra; y una breve entrevista semi-dirigida que exploró cual es el apoyo más valorado y el que se necesita con mayor frecuencia. Los datos fueron analizados de manera cualitativa mediante la generación de categorías y, posteriormente, de manera cuantitativa, utilizando paquetes estadísticos informatizados para Ciencias Sociales. Los resultados evidencian que el apoyo emocional es el más valorado $(72 \%)$ y más necesitado $(37,5 \%)$ en todas las franjas etarias.
\end{abstract}

Palabras Clave: apoyo social, curso vital, apoyo valorado, apoyo necesitado.

\section{Assesment of social support during vital course}

\begin{abstract}
Social support is a multidimensional construct that has a structural perspective and a functional perspective. From the structural perspective, the notion of social network is emphasized, which corresponds to the bonds that a person possesses and what they perceive as significant. The functional perspective refers to the functions performed by this network, the support it provides, and its effects on the well-being of the individual. The networks are modified throughout life, in terms of the size and composition of their bonds, which may affect the support it grants. For this reason, the objective of this research was to explore the most valued and needed supports during the life course. For this purpose, a transverse, descriptive, non-experimental design with a combination of quantitative and qualitative methodology was implemented. The sample was constituted by 120 individuals of both sexes, 30 by age group ( 20 to 30,40 to 50, 60 to 70 and 80 to 90) residents of the city of Mar del Plata. To the total of the sample they were administered: a questionnaire of sociodemographic data that allowed to characterize the sample; and a brief semi-directed interview that explored which is the most valued and most frequently needed support. The data was analyzed in a qualitative manner by the categories of each generation, and then quantitatively using computerized statistical packages for Social Sciences. The results showed that emotional support is the most valued $(72 \%)$ and most needed $(37.5 \%)$ in all age groups.
\end{abstract}

Keywords: social support, life course, valued support, support needed.

1 Y 2 Universidad Nacional de Mar del Plata, Argentina; enriquelombardo2014@gmail.com 
El concepto de apoyo social durante los últimos años ocupa un lugar central en la literatura científica por la incidencia que, como variable, ha demostrado tener sobre la salud y el bienestar de las personas (Aguerre \& Bouffard \& Curcio, 2008; Cassel, 1974; 1976; Caplan 1974; 1976; Coob 1976; Fernández, Clúa, Báez \& Ramírez, 2000; Gottlieb, 1981). Este interés se refleja en el creciente número de investigaciones y publicaciones referidas al tema y la amplia variedad de programas de prevención y de intervención formulados desde este modelo teórico.

Existen diversas definiciones de apoyo social. Fernández Ballesteros, Izal, Montorio, González y Díaz Veiga (1992) lo conceptualiza como la ayuda, ya sea emocional, instrumental o de otra indole, que se deriva de un determinado entramado social. Thoits (1983) lo define como el grado en que las necesidades sociales básicas (afiliación, afecto, identidad, seguridad, y aprobación) de la persona son satisfechas a través de la interacción con otros. Andrade y Vaitsman (2002) refieren que el apoyo social consiste en las relaciones de intercambio, que implican obligaciones mutuas y lazos de dependencia mutua que pueden ayudar a crear el sentido de la coherencia y el control de la vida, que benefician a la salud de las personas. Todas estas definiciones señalan las relaciones sociales que, de forma natural, configuran el entorno del sujeto y con las cuales interactúa.

El apoyo social es un constructo multidimensional que posee una perspectiva estructural y una perspectiva funcional. Desde la perspectiva estructural, se enfatiza la noción de red social, que corresponde a los vínculos que una persona posee. Por lo tanto, pueden ser identificados y cuantificados. La perspectiva funcional hace referencia a las funciones que desempeña esa red, los apoyos que proporciona y sus efectos en el bienestar del sujeto. Dentro de las funciones de la red se incluye: la compañía social, el apoyo emocional, guía cognitiva y consejos y ayuda económica, de servicios y acceso a nuevos contactos (Sluzki, 1996).

Cercanas a esta perspectiva, Kahn y Antonucci (1980) plantean la noción de convoy como un conjunto de roles y compañias que participan en el sostén de un sujeto a lo largo de su vida. Esta noción sostiene que las personas ocupan y abandonan roles en los distintos momentos de su vida y cada rol está acompañado por determinadas relaciones. Por ejemplo, un estudiante universitario seguramente habrá incorporado a compañeros de estudio en su red, mientras que, en la adultez, la actividad laboral favorecerá la inclusión de compañeros del trabajo. De esta manera, las redes son dinámicas y pueden modificarse a lo largo de la vida, como también en cada etapa vital, tanto en relación con el tamaño como en la composición de sus vínculos, lo que puede repercutir en el apoyo que proporciona. Si bien existen numerosas investigaciones que indagaron las características estructurales de las redes (su tamaño) (Arias, 2004; Arias, Botas \& Polizzi, 2011; Cornwell, Schumm, Laumann, \& Graber, 2009; Fernández del Valle \& Bravo, 2000; Ham, 2002; Huenchuán \& Sosa, 2002); y sus características funcionales (los vínculos que proporcionan apoyos y los apoyos brindados) (Arias, 2004, 2013; Barros, 2002; Arriagada Mellado, 2012) existe relativamente poca investigación acerca de los apoyos más valorados y necesitados de acuerdo con cada etapa del curso vital. Por este motivo, el propósito del presente trabajo es exponer los resultados de una investigación que tenía como objetivo identificar el apoyo más valorado y el más frecuentemente necesitado en cuatro grupos etarios. Dada la importancia de las redes de apoyo por su incidencia en el bienestar de 
los sujetos, tanto por el mejoramiento de las condiciones objetivas de vida mediante la provisión de apoyos materiales e instrumentales como por el impacto significativo que brinda el apoyo emocional (Huenchuan, Guzmán, \& Montes de Oca, 2003), consideramos necesario incluir personas que transiten distintas etapas evolutivas: la juventud, la adultez y la vejez, tanto incipiente como avanzada, de modo de incrementar los conocimientos sobre el apoyo social a lo largo del desarrollo de un individuo. Y también disponer de herramientas interventivas que permitan fortalecer las redes de apoyo en distintas etapas vitales.

\section{Antecedentes sobre apoyo social}

Existen diversas disciplinas que plantean el surgimiento del apoyo social como un tema de interés dentro de su campo de estudio. Madariaga Orozco, Abello Llanos y Sierra García (2003) sostienen que el estudio de las redes sociales tiene su origen en los años cuarenta; luego, en los años setenta, alcanza un desarrollo interesante en la sociología y la antropología, para extenderse posteriormente a todo el campo de las ciencias sociales. Arias (2004) menciona que este modelo teórico surge de modelos ecológicos que intentaban comprender y explicar la conducta humana en función de la relación dialéctica entre el individuo y su ambiente. Gracia Fuster (1997) plantea que en el campo de la Psicología Social en la década del 60, se encuentran varias investigaciones sobre la temática que demostraban cómo, ante situaciones de temor o ansiedad, las personas tienden a buscar la compañia de otras, y que esta compañia además tiene efectos frente a situaciones estresantes. Villalba Quesada (1993) menciona que el concepto de apoyo social surge cuando existe una asociación entre problemas de salud mental y variables sociales como desintegración social, movilidad geográfica o estatus matrimonial, en donde el elemento común de esas variables situacionales era la ausencia de lazos sociales adecuados o la disrupción de las redes sociales. Por su parte, Cassel, Cobb y Caplan en los años 70, a partir de hallazgos científicos, plantearon que el apoyo social protege a las personas de las consecuencias negativas, físicas y psicológicas de sucesos estresantes. Esta propuesta la sustentaron en sus investigaciones experimentales, en la que tanto animales como humanos que se encontraban en compañía de otros significativos o iguales, expuestos a estímulos estresantes, no sufrian efectos adversos, a diferencia de otros sujetos en condiciones de aislamiento. De esta manera, Caplan (1974) enfatiza el apoyo social como una función básica de la red social que tiende a mantener la integridad física y psicológica del individuo.

Otro planteo interesante es el que realizan Guzmán et al. (2003) cuando diferencian el contexto de desarrollo de este concepto delimitando dos corrientes, una anglosajona y otra latinoamericana. En la tradición anglosajona, destacan los trabajos de Lopata (1979), Cobb (1976), Walker (1977) y Maguire (1980) entre otros, que conceptualizan la red como proveedora de recursos y apoyos que generan beneficios y efectos en la conducta de los individuos. En América Latina, destacan fuertemente los trabajos de la antropología respecto de las redes sociales. Su énfasis se centró en la importancia de las redes sociales en las estrategias de reproducción social de aquellos sujetos o colectivos que se encontraban en situación de vulnerabilidad social. Los trabajos de Lomnitz (1977), Abello, Madariaga Orozco y Hoyos de los Ríos (1997), Enriquez-Rosas (2008), Montero (2003), 
Pasarin (2009), Ávila-Toscano (2009), demuestran el papel relevante que cumplen las redes en situaciones de pobreza o desamparo social. En Argentina, Dabas (1993) desarrolla el estudio de las redes mediante un dispositivo que denominó multi-familias, y que aplicó tanto en instituciones educativas, de salud y jurídicas como en programas juveniles y organizaciones barriales. Sus resultados generaron el trabajo y desarrollo de redes comunitarias que se organizan para dar solución a demandas sociales especificas.

De esta manera, existen diferentes autores que han definido el concepto de apoyo social como también diversas líneas de investigación a partir de la consideración de distintas variables que lo determinan, sean estas la integración social, los recursos materiales o sociales, los vínculos sociales, el bienestar o mejora de la salud. En todos los casos, está implícita la idea de relaciones interpersonales, de vínculos y de intercambio emocional y material como parte del apoyo social, el cual se ofrece a los sujetos si se producen dificultades, crisis o conflictos que puedan afectarlos.

\section{Caracterización de las redes de apoyo}

Las redes sociales son sistemas abiertos, constituyen un proceso permanente de construcción que se da a nivel individual y colectivo (Dabas, 1993). Dado que acompañan el curso vital de un sujeto, están en perpetuo flujo, cambian su configuración, muestran diversidad de afluentes y admiten itinerarios singulares de acuerdo con el momento vital y contextual del sujeto. Slusky (1996) considera a la red social personal como la suma de todas las relaciones que un individuo percibe como significativas. En concordancia con los hallazgos de la psicología positiva, se plantea que las buenas relaciones con otras personas son el factor que contribuye de manera más importante a la buena vida psicológica. Las buenas relaciones proporcionan apoyo emocional e instrumental en momentos de estrés y desafio, pero también proporcionan un sentido de conexión y la oportunidad de celebrar las cosas buenas de la vida (Park, Peterson \& Sun, 2013). Por este motivo, disponer de apoyo social tiene una especial relevancia para la salud, adaptación e integración social y bienestar.

Pinazo Hernandis y Sánchez (2005) distinguen tres ámbitos como posibles fuentes del apoyo social, es decir, en los que se produce o puede producirse el apoyo: la comunidad, las redes sociales y las relaciones intimas o relaciones interpersonales. Cada uno de los distintos estratos de las relaciones sociales conlleva vinculos distintos entre los individuos y su entorno social con características y connotaciones diferentes:

a) En el nivel macrosocial o comunitario, la persona se identifica y participa en el entorno social. Se destaca como indicador del sentido de pertenencia.

b) A través del nivel medio, mesosocial o de las redes sociales, se accede directa e indirectamente a un número relativamente amplio de personas. Es indicador del sentimiento de vinculación.

c) El nivel microsocial, o de las relaciones de intimidad y confianza, es el estrato más significativo del sujeto. Implica un sentimiento de compromiso ya que se producen intercambios mutuos y recíprocos y se comparte un sentido de responsabilidad por el bienestar del otro.

Desde una perspectiva funcional, vínculos $\mathrm{y} / \mathrm{o}$ relaciones cubren distintas necesidades y demandas del sujeto, ya sea en el plano afectivo, 
material y/o informacional (Pinazo Hernandis, 2006). El tipo de apoyo más útil dependerá del problema con el que se encuentre el sujeto y por el cual necesita ayuda o sentirse apoyado. Por ejemplo, la aparición de una enfermedad puede requerir varios tipos de apoyo; la pérdida de un ser querido puede requerir apoyo emocional, pero después son necesarias las relaciones sociales para integrarse o reinsertarse socialmente. Sluzki (1996) incluye como funciones de la red a la compañia social, que alude a la ejecución de actividades conjuntas o simplemente sentirse junto a otros; el apoyo emocional, que hace referencia al disponer de alguien con quien compartir sentimientos profundos e intimos, esto es, poder contar con el compromiso emocional y la buena voluntad del otro, en lo que se juega el amor, el cariño y la comprensión, entre otras emociones. A esto se suma la guía cognitiva y consejos, que alude a las interacciones que tienen como fin compartir información personal o social, aclarar expectativas y proveer modelos de desempeño de rol; y la ayuda económica, material y/o de servicios, que hace referencia a la colaboración específica (puede ser la ayuda en actividades del hogar, el cuidado y acompañamiento). Y por último, el acceso a nuevos contactos se refiere a la posibilidad de conexión con otras personas y redes que hasta entonces no eran parte de la red del individuo.

Existen pocas investigaciones que hayan estudiado cuál es el apoyo más valorado en el curso vital. Hay evidencias empíricas sobre los vínculos que son fuentes de apoyo. Por ejemplo, Pinazo Hernandis (2006) sostiene que la mayor parte de las personas reciben el apoyo más importante a través de las redes informales. Kahn y Antonucci (1980) encontraron que a lo largo del curso vital las personas atraviesan diversas crisis o momentos de cambio, en los que están presentes un número reducido de personas, familiares y amigos, que brindan apoyo emocional e instrumental. Si bien las redes son dinámicas y cambiantes, de acuerdo con esta teoría, también mantienen cierta estabilidad. En este sentido, también existen investigaciones que señalan que las personas en condición de riesgo que se sienten apoyadas por sus amigos y familiares tienen menor probabilidad de presentar consecuencias adversas para su salud y mantienen un mayor ajuste psicológico (Gracia Fuster \& Herrero, 2006). En un estudio realizado en los principales centros urbanos de la Argentina se mostró que el $70 \%$ del total de las personas encuestadas mantenía lazos de ayuda emocional que le permitían compartir sus problemas con los demás. En los estratos más bajos se involucran menos que en los sectores más altos (67\% en el nivel muy bajo y bajo, mientras en el sector medio-alto llega al $80 \%$ (Lépore, \& Lagarde, 2006). En el informe sobre la Encuesta de la Deuda Social (Universidad Católica Argentina, 2016), se destaca la ausencia de red social, es decir, la ausencia de vínculos de apoyo. Esto se pone de manifiesto en el hecho de que 1 de cada 4 encuestados respondió no tener alguien que lo ayude frente a sus problemas (Rodríguez Espinola, 2016).

Diversos estudios evidencian que la cantidad de vínculos íntimos y el apoyo emocional del cual se dispone son relativamente estables a lo largo de la vida y hasta en edades más avanzadas (Antonucci, 2001; Cornwell, Schumm, Laumann, \& Graber, 2009; Wrzus, Hänel, Wagner, \& Neyer, 2013). Como también existen hallazgos que demuestran que vivir con familiares en vez de solos genera mayor grado de salud mental, física y emocional en la vejez (Bozo, Toksabay \& Kürüm, 2009; Vivaldi \& Barra, 2012).

En lo que respecta a los vínculos que son fuentes de apoyo, varias investigaciones mostraron que entre los apoyos que recibe el adulto mayor, el 
cónyuge y los hijos/as son los principales proveedores de los mismos (Castellano-Fuentes, 2014; Pelcastre-Villafuerte, Treviño-Siller, GonzálezVázquez \& Márquez-Serrano, 2011; Polizzi \& Arias, 2014; Vera Noriega, Sotelo Quiñonez \& Dominguez Guedeas, 2005). Barros (1994) plantea que existen diferencias según nivel socioeconómico: en los estratos bajos la mejor calificación la obtiene el cónyuge o la pareja, mientras que en los estratos medios corresponde a los hijos. También sostiene que los amigos/as constituyen una fuente secundaria de apoyo, a quienes se recurre en busca de apoyo cognitivo, y tienen mayor importancia en aquellas personas que tienen escasas relaciones familiares (Barros, 2002). Polizzi y Arias (2013) en una investigación en la que indagaron cuáles eran los vínculos de la red de apoyo que brindaban más satisfacción en adultos mayores, plantean que los hijos y los amigos son los vínculos que brindan mayor satisfacción, y destacan también que los apoyos más valorados por los adultos mayores son el emocional y la compañía social. Montes de Oca (2002) realizó una investigación en México donde uno de sus objetivos era identificar las formas de apoyo que dan y reciben los adultos mayores. En sus resultados señala que las mujeres adultas mayores aportan servicios, trabajo, hacen visitas a enfermos y realizan compañía. Los varones, por su parte, brindan ayuda, enseñan o proporcionan compañia. Por último, con respecto a los apoyos más necesitados durante el curso vital, Pinazo Hernandis (2006), en referencia a las personas mayores, sostiene que en edades avanzadas, a partir de los 80 años, la persona mayor comienza a experimentar una dependencia acentuada a partir de la cual necesita de personas para la realización de las actividades cotidianas necesarias. Si bien la mayor parte del cuidado de los mayores lo siguen realizando las propias familias, también crece la importancia de las personas contratadas como cuidadores.

\section{METODOLOGÍA}

Se implementó un diseño no experimental, de tipo transversal/descriptivo. Se utilizaron métodos mixtos combinando la exploración cualitativa y cuantitativa. Se seleccionó una muestra no probabilistica intencional que estuvo constituida por 120 sujetos pertenecientes a distintas etapas vitales: 30 de cada grupo de edad (20 a 30, 40 a 50, 60 a 70 y 80 a 90), residentes de la ciudad de Mar del Plata. Cada uno de los grupos se equiparó por género (15 varones y 15 mujeres). Al total de la muestra se les administró en un único encuentro de manera oral e individual: un cuestionario de datos sociodemográficos que, mediante preguntas abiertas y cerradas, exploró variables de base que permitieron caracterizar la muestra como género, edad, tipo de hogar, conformación de grupo conviviente, nivel de instrucción y ocupación. En segundo lugar, una breve entrevista semi-dirigida que exploró entre los cinco apoyos (emocional, social, practico, financiero o consejo/guía) cuál es el apoyo que más valora y cuál el que necesita con mayor frecuencia. Antes de responder a estas preguntas, se daba información sobre cada uno de estos apoyos, explicitando ejemplos específicos de acuerdo con cada grupo de edad sobre lo que proporciona ese apoyo. La recolección de los datos se realizó en lugares públicos, en organizaciones de salud, educativas o laborales con las cuales se han establecido convenios previos que facilitaron el acceso a las mismas.

Durante el proceso de recolección de datos, las personas que formaron parte de la muestra firmaron el Consentimiento Informado luego de haber 
recibido las explicaciones pertinentes. En este se explicitan los objetivos de la investigación y se aclara que la participación es voluntaria, anónima, confidencial y que la información revelada se utilizará con fines exclusivamente científicos, bajo la jurisdicción de la Ley Nacional 25.326 de protección de los datos personales. Los datos recolectados mediante el cuestionario fueron analizados de manera cuantitativa utilizando paquetes estadísticos informatizados para Ciencias Sociales. Los datos resultantes de la entrevista se analizaron en primer lugar de forma cualitativa, mediante análisis de contenido y asignación de categorias. Luego de su categorización, se procedió a un análisis estadístico, utilizando el programa $R$, versión 3.4.1 y se realizaron distribuciones de frecuencias y porcentajes.

\section{RESULTADOS}

Los resultados indican que el apoyo emocional es el más valorado por todos los grupos etarios (72.2\%). La valoración más alta la reporta el grupo que oscila entre los 40 y 50 años (82\%) y la más baja (60\%) el grupo de 80 a 90 años. Posteriormente, el segundo apoyo más valorado varía según grupo de edad, por ejemplo, para los grupos de 20 a 30 y 60 a 70 años, el apoyo social se encuentra en segundo lugar y consejo en tercer lugar; y para el grupo de 40 a 50 y 80 a 90, el consejo se encuentra como el segundo apoyo más valorado y en tercer lugar el apoyo social. Por otra parte, tal como se observa en la tabla 1, el apoyo práctico y financiero no se menciona para los grupos de 40 a 50 y 60 a 70 años; y en los adultos mayores de 80 a 90 años aparecen todos los apoyos valorados, al igual que en el grupo de 20 a 30, pero con menores frecuencias. Finalmente, para el grupo de los más jóvenes, el apoyo emocional es el más valorado, mientras que el práctico y el financiero son menos valorados.

Tabla 1.

Tipos de apoyo más valorados según grupo de edad.

\begin{tabular}{lcccccccccc}
\hline \multirow{2}{*}{$\begin{array}{l}\text { Tipo de } \\
\text { apoyo }\end{array}$} & \multicolumn{2}{c}{$\mathbf{2 0} \mathbf{~ a ~} \mathbf{3 0}$} & \multicolumn{2}{c}{$\mathbf{4 0}$ a $\mathbf{5 0}$} & \multicolumn{2}{c}{$\mathbf{6 0}$ a $\mathbf{7 0}$} & \multicolumn{2}{c}{$\mathbf{8 0}$ a $\mathbf{9 0}$} & \multicolumn{3}{c}{ Total } \\
\cline { 2 - 12 } & $\boldsymbol{n}$ & $\mathbf{\%}$ & $\boldsymbol{n}$ & $\mathbf{\%}$ & $\boldsymbol{n}$ & $\mathbf{\%}$ & $\boldsymbol{n}$ & $\mathbf{\%}$ & $\boldsymbol{n}$ & $\mathbf{\%}$ \\
\hline Emocional & 21 & 70 & 23 & 82.1 & 19 & 76 & 15 & 60 & 78 & 72.2 \\
Social & 4 & 13.3 & 1 & 3.6 & 4 & 16 & 2 & 8 & 11 & 10.2 \\
Práctico & 1 & 3.3 & 0 & 0 & 0 & 0 & 2 & 8 & 3 & 2.8 \\
Financiero & 1 & 3.3 & 0 & 0 & 0 & 0 & 3 & 12 & 4 & 3.7 \\
Consejo & 3 & 10 & 4 & 14.3 & 2 & 8.0 & 3 & 12 & 12 & 11.1 \\
\hline Total & 30 & 100 & 28 & 100 & 25 & 100 & 25 & 100 & 108 & 100 \\
\hline
\end{tabular}

Nota: El Total de cada grupo de edad no alcanza en algunos casos a $n=30$, ya que algunos entrevistados no respondieron la pregunta correspondiente.

Los resultados en relación con el objetivo que pretendia identificar los tipos de apoyo más necesitados, según grupo de edad, indican que el mayor porcentaje corresponde al apoyo emocional (37.5\%), seguido por el social $(27 \%)$, en tercer lugar el práctico (19\%), luego consejo (17\%) y finalmente el financiero (9\%) para el total de la muestra. En el caso del grupo de 80 a 90, la frecuencia es igual en el emocional y el social. En el resto de los grupos, el apoyo social fue el segundo más frecuentemente necesitado, con excepción del grupo de 40 a 50 en el que el práctico ocupó el segundo lugar. En cuanto a la distribución de los porcentajes, el grupo de 60 a 70 años fue quien reportó necesitar con mayor frecuencia el apoyo emocional (57\%). El grupo de 80 a 90 
años fue de todos los grupos etarios el que menos necesita de este apoyo. La ayuda práctica es un apoyo que se presenta en el grupo de 40 a 50 en segundo lugar y en los adultos mayores de 80 a 90 en tercer lugar. Los grupos de 20 a 30, de 40 a 50 y 60 a 70 eligieron consejo y guía en tercer lugar. E1 apoyo financiero ocupó el cuarto lugar en todos los grupos con porcentajes bajos: iguales o menores a $10 \%$ (ver tabla 2 ).

Tabla 2.

Tipos de apoyo que necesitan más frecuentemente según grupo de edad.

\begin{tabular}{lcccccccccc}
\hline \multirow{2}{*}{$\begin{array}{l}\text { Tipo de } \\
\text { apoyo }\end{array}$} & \multicolumn{2}{c}{$\mathbf{2 0}$ a $\mathbf{3 0}$} & $\mathbf{4 0}$ a $\mathbf{5 0}$ & $\mathbf{6 0}$ a $\mathbf{7 0}$ & $\mathbf{8 0}$ a $\mathbf{9 0}$ & \multicolumn{2}{c}{ Total } \\
\cline { 2 - 11 } & $\boldsymbol{n}$ & $\mathbf{\%}$ & $\boldsymbol{n}$ & $\mathbf{\%}$ & $\boldsymbol{n}$ & $\mathbf{\%}$ & $\boldsymbol{n}$ & $\mathbf{\%}$ & $\boldsymbol{n}$ & $\mathbf{\%}$ \\
\hline Emocional & 10 & 33 & 10 & 34 & 17 & 57 & 8 & 29 & 45 & 37.5 \\
Social & 9 & 30 & 4 & 14 & 6 & 20 & 8 & 29 & 27 & 22.5 \\
Práctico & 3 & 10 & 8 & 28 & 2 & 7 & 6 & 21 & 19 & 15.8 \\
Financiero & 3 & 10 & 2 & 7 & 2 & 7 & 2 & 7 & 9 & 7.0 \\
Consejo & 5 & 17 & 5 & 17 & 3 & 10 & 4 & 14 & 17 & 14.2 \\
Ninguno & 0 & 0 & 1 & 3 & 0 & 0 & 2 & 7 & 3 & 2.5 \\
\hline Total & 30 & 100 & 29 & 100 & 30 & 100 & 28 & 100 & 120 & 100 \\
\hline Nota: El Total de cada grupo de edad no alcanza en algunos casos a $n=30$, ya que algunos \\
entrevistados no respondieron la pregunta correspondiente.
\end{tabular}

\section{CONCLUSIONES}

Los resultados de este estudio señalan al apoyo emocional como el más valorado y más necesitado en todas las franjas etarias. Por tanto, es posible inferir que el apoyo emocional resulta central en la construcción de redes sociales a lo largo del curso de la vida.

El apoyo social fue el segundo apoyo más valorado y que refirieron necesitar más frecuentemente, también en segundo lugar, los sujetos consultados. Nuestro estudio parece corroborar la fuerte tendencia a considerar los aspectos emocionales y afectivos por sobre los instrumentales y cognitivos en la valoración de los tipos de apoyo. En este sentido encontramos consistencia entre nuestros resultados con los de Kahn y Antonucci (1980). Es posible advertir que la valoración de los apoyos está relacionada con los roles de cada etapa de la vida, tal como lo plantean estos autores. Si bien el apoyo emocional es el más valorado y necesitado por todos los grupos, también hay que señalar que hay apoyos más necesitados de acuerdo con la etapa por la que transita el sujeto. En la mediana edad, por ejemplo, que es una etapa donde se conjugan la crianza de los hijos, el trabajo y en algunos casos el cuidado de los padres, la ayuda práctica es muy requerida. También lo es para los mayores de 80 años, en donde, por razones que pueden tener que ver con mayor dependencia, se necesite disponer de este apoyo. Estos resultados se corresponden con lo planteado por Pinazo Hernandis (2006), que sostiene que a partir de los 80 años las personas necesitan ayuda para la realización de las actividades cotidianas necesarias.

Los apoyos que se necesitan con mayor frecuencia presentan mayor variabilidad en todos los grupos etarios. Sin embargo, respecto a su valoración, el apoyo emocional presenta diferencias frente al resto, lo que se debe a su componente subjetivo, es decir, a la creencia en la importancia de disponer de él. Estos hallazgos nos permiten pensar que los sujetos construyen sus redes priorizando vínculos emocionales (positivos) por sobre aquellos que son mayormente proveedores de insumos materiales $y$ 
simbólicos, más allá de que los necesiten. Los tipos de apoyo instrumental y cognitivo (económico y consejo y guía) no parecen ser promotores de la ampliación y enriquecimiento de la red en ninguna etapa de la vida. A partir de la construcción de un ambiente afectivo emocional, se forman y enriquecen las redes de apoyo emocional, social e instrumental. La satisfacción de las necesidades materiales y cognitivas se lleva a cabo, inicialmente, dentro del entorno afectivo emocional constituido. Si en ese entorno no se puede satisfacer la necesidad, por carencias de quienes forman parte de la red o por la pobreza de la red misma, la búsqueda se extenderá fuera de ese entorno, en figuras que resultan menos significativas.

No podemos pensar un sujeto por fuera de una red en la que resulte al mismo tiempo un apoyo para otros y que estos sean, de manera recíproca, un apoyo para él. El sujeto necesita y busca ocupar un lugar en un sistema social como la familia, el grupo de amigos, o un grupo de trabajo, y busca ser reconocido en ese contexto. Esta búsqueda es transversal a lo largo del curso de la vida. En ese ámbito se plantean las interacciones que implican algún tipo de ayuda. Inicialmente, suponemos que en distintos momentos de la vida cobran importancia unas $u$ otras fuentes de apoyo social en función de características evolutivas o situaciones circunstanciales. Sin embargo, emerge el interrogante de por qué resulta tan importante el apoyo emocional en todas las etapas de la vida.

El presente estudio, como otras investigaciones sobre redes de apoyo, nos indica la existencia de un núcleo fuertemente ligado al aspecto emocional como necesidad básica. Todo sujeto en su curso vital seguramente tendrá cambios en su forma de vida, en sus relaciones interpersonales y con el medio. Situaciones que pueden generar momentos dificiles y para los cuales se necesitará la compañia de otros que brinden contención, seguridad y tranquilidad. La presencia de vínculos que estén disponibles cuando se precisan parecería que es una necesidad, independientemente de la edad que tenga el sujeto. El apoyo emocional fortalece la capacidad del sujeto para hacer frente en forma más efectiva a las demandas de la vida diaria y a los problemas que conlleva. Contar con este apoyo incrementa la percepción que se tiene de sí mismo, la autoestima, el bienestar subjetivo y tiene efectos en una buena salud. Comprender la importancia de disponer de apoyo emocional nos plantea el desafio de desarrollar intervenciones dirigidas a fortalecer las redes de apoyo de las personas durante el curso vital.

\section{Alcances y Limitaciones}

Adquiere relevancia dimensionar la importancia que tiene el estudio y conocimiento sobre el apoyo social. Si bien los resultados de esta investigación no pueden ser generalizados porque la muestra es reducida, evidencian la importancia que tiene para los sujetos disponer de apoyo emocional independientemente su edad. Esto señala que poseer redes amplias y suficientes, con vínculos que puedan satisfacer variadas funciones de apoyo, resulta un factor que tiene una alta incidencia en el bienestar subjetivo a lo largo de la vida y reviste una particular importancia en el proceso de envejecimiento. Los resultados de este estudio aportan nuevos datos de interés, ya que hasta el momento hay poca evidencia acerca de los apoyos valorados y necesitados en las distintas franjas etarias y, en especial, en adultos mayores de edad avanzada. Resultaría interesante profundizar este trabajo ampliando la muestra, para poder corroborar la consistencia de estos 
resultados, como también, la posibilidad de realizar estudios longitudinales que puedan explorar la modificación o no de los apoyos valorados y necesitados a través de los años y arribar a nuevos hallazgos de importancia para el conocimiento del curso vital.

\section{REFERENCIAS}

Abello, R., Madariaga, C. \& Hoyos, O. (1997). Redes sociales como mecanismode supervivencia: en estudio de casos en sectores de extrema pobreza. Revista Latinoamericana de Psicología, (29) 115-137.

Aguerre, C., Bouffard, L., \& Curcio, C. (2008). Envejecimiento exitoso: Teorias, investigaciones y aplicaciones clínicas. Revista de la Asociación Colombiana de Gerontología y Geriatría, 22(2), 11461162.

Andrade, G. R. B. \& Vaitsman, J. (2002). Apoyo social e redes: conectando solidariedade saúde. CiêncSaúdeColetiva, 7(4), 925-34.

Antonucci, T. C. (2001). Social relations: An examination of social networks, social support. Handbook of the psychology of aging, 3, 427-453.

Arias, C. (2004). Red de Apoyo Social y Bienestar Psicológico en Personas de Edad. Mar del Plata, Argentina: Suárez.

Arias, C. (2013). El apoyo social en la vejez: la familia, los amigos y la comunidad. Revista Kairós Gerontología, 16(6), 25-40.

Arias, C. Botas, N. \& Polizzi, L. (abril, 2011). Caracteristicas de lared de apoyo social: comparación entre adultos jóvenes yadultos mayores residentes en la ciudad de Mar del Plata. Ponencia presentada en el XI Congreso Virtual de Psiquiatria Interpsiquis.

Arriagada Mellado, M. (2012). Apoyo emocional, instrumental y material hacia eladulto mayor: una percepción desde los hijos (Tesis de maestría no publicada). Universidad del BíoBío, Chile.

Ávila-Toscano, J. (2009). Redes sociales, generación de apoyo socialante la pobreza y calidad de vida. Revista Iberoamericana de Psicología: Ciencia y Tecnologia, 2(2), 65-73.

Barros C. (1994). Apoyo social y bienestar del adulto mayor. Documento Instituto de Sociología, 60.

Barros, C. (2002). Relaciones e intercambios familiares del adulto mayor. Trabajo presentado en el IV Congreso Chileno de Antropología, Santiago de Chile.

Bozo, Ö., Toksabay, N. E., \& Kürüm, O. (2009). Activities of daily living, depression, and social support among elderly Turkish people. The Journal of psychology, 143(2), 193-206. https://doi.org/10.3200/JRLP.143.2.193-206

Fernández del Valle J. \& Bravo, A. (2000). Estructura y dimensiones de apoyo en la red social de los adolescentes. Anuario de Psicología, 31(2), 87-105.

Caplan, G. (1974). Systems and Community Mental Health. New York: Behavioral Publications. https://doi.org/10.2190/WF7X-Y1LO-BFKH-9QU2

Caplan, G. (1976). The family as support system. En Caplan,G. \& Killilea, M. (Eds.). Support systems and mutual help: multidisciplinary explorations (pp. 19-36). New York: Grune y Stratton. https://doi.org/10.1093/oxfordjournals.aje.a112281

Cassel, J. (1974). Psychosocial processes and stress: theoretical formulations. International Journal of Health Services, 6, 471-482.

Cassel, J. (1976). The contribution of the social environment to host resistance. American Journal of Epidemiology, 104, 107-123.

Castellano Fuentes, C. (2014). La influencia del apoyo social en elestado emocional y las actitudes hacia la vejez y el envejecimiento en una muestra de ancianos. International Journal of psychology and psychological therapy, 14(3), 365-377.

Cobb, S. (1976). Social support as a moderator of life stress. Psychosomatic Medicine, 38, 300-314. https://doi.org/10.1097/00006842-197609000-00003

Cornwell, B., Schumm, L. P., Laumann, E. O. \& Graber, J. (2009). Social Networks in the NSHAP Study: Rationale Measurement and preliminary Findings. Journal of Gerontology: Social Sciences, 64(1), 4755. https://doi.org/10.1093/geronb/gbp042

Dabas, E. (1993). Red de redes. Las prácticas de la intervención en redes sociales. Buenos Aires: Paidós.

Enríquez-Rosas, R. (2008). Redes sociales y de apoyo emocional en mujeres pobres urbanas. En R. Enriquez-Rosas (Ed.), El crisol de la pobreza. Mujeres, subjetividades, emociones y redes sociales (pp. 313-336). Jalisco: ITESO.

Fernández, N., Clúa, A., Báez, R., \& Ramírez, M. (2000). Estilos de vida, bienestar subjetivo y salud de los ancianos. Revista Cubana de Medicina General,(16) 6-12.

Fernández Ballesteros, R., Izal, M., Montorio, I., González, J. L., \& Diaz Veiga, P. (1992). Evaluación e intervención psicológica en la vejez. Barcelona: Martinez. Roca.

Gottlieb, B. (1981). Social networks and social support. Studies in Community Mental Health, 4, 304-310.

Gracia Fuster, E. (1997). El Apoyo Social en la Intervención Comunitaria. Buenos Aires: Paidos.

Gracia Fuster, E. \& Herrero, J. (2006). La comunidad como fuente de apoyo social: evaluación e implicaciones en los ámbitos individual y comunitario. Revista Latinoamericana de Psicología, 38(2), 327-342.

Ham, R. (diciembre, 2002). Calidad de vida y redes de apoyo social de las personas en edades avanzadas en Ciudad de México. Trabajo presentado en la Reunión de Expertos en Redes de Apoyo Social a Personas Mayores, CEPAL, Santiago de Chile

Huenchuán, S., Guzmán, J. M., \& Montes de Oca, V. (2003). Redes de apoyo social de las personas 
mayores: marco conceptual. Notas de población, 29(77), 35-70.

Huenchuán, S. \& Sosa, Z. (diciembre, 2002) Calidad de vida y redes de apoyo social de personas mayores en Chile. Trabajo presentado en la Reunión de Expertos en Redes de Apoyo Social a Personas Mayores, Santiago de Chile.

Kahn R. L., Antonucci T. C. (1980). Convoys over the life course: Attachment, roles, and social support. En Baltes P. B., Brim O, editors. (Eds.), Life-span development and behaviour (3) 254-283. New York: AcademicPress.

Lépore, S., Lagarde, C. (2006). Necesidades afectivas y de apoyo emocional. Programa Observatorio de la Deuda Social Argentina, 7, 1-6.

Lomnitz, L. (1977). Cómo sobreviven los marginados. México, D.F.: Editorial Siglo XXI.

Lopata, H. (1979). Women As Widows: Support Systems. Nueva York: Elsevier.

Madariaga Orozco, C., Abello Llanos, R. \& Sierra García, O. (2003). Redes sociales. Infancia, familia y comunidad. Barranquilla: Uninorte.

Maguire, L. (1980). The interface of social workers with personal networks. Social Work with Groups, 3, 39 49. https://doi.org/10.1300/J009v03n03_04

Montero, M. (2003). Teoría y práctica de la Psicología Comunitaria. La tensión entre comunidad y sociedad. Buenos Aires: Paidós

Montes de Oca, V. (diciembre, 2002). Participación, organización y significado de las redes de apoyo comunitario entre las mujeres adultas mayores. La experiencia de la Colonia de Aragón en la Delegación Gustavo A. Madero, Ciudad de México. Trabajo presentado en la Reunión de Expertos en Redes de Apoyo Social a Personas Mayores, Santiago de Chile.

Park, N., Peterson, C., \& Sun, J.K. (2013). Positive Psychology: Research and practice. Terapia psicológica, 31(1), 11-19. https://doi.org/10.4067/S0718-48082013000100002

Pasarin, L. (2009). Estudio antropológico de redes sociales de madres adolescentes durante el embarazo. Avá, 14, 1-15.

Pelcastre-Villafuerte, B., Treviño-Siller, S., González-Vázquez, T., \& Márquez-Serrano, M. (2011). Apoyo social y condiciones de vida de adultos mayores que viven en la pobreza urbana en México. Cadernos de Saúde Pública, 27(3), 460-470. https://doi.org/10.1590/S0102-311X2011000300007

Pinazo Hernandis, S. (2006). Relaciones Sociales. En C. Triado \& F. Villar (Eds.), Psicología de la Vejez (pp. 253-286). Madrid: Alianza Editorial.

Pinazo Hernandis, S. \& Sánchez M. (2005). Gerontología: Actualización, innovación y propuestas. España: Pearson Prentice Hall.

Polizzi, L., \& Arias, C. J. (2014). Vínculos que brindan más satisfacción en la red de apoyo social de adultos mayores. Pensando Psicología, 10(17), 61-70.

Rodríguez Espínola, S. (2016). Situación de la salud y condiciones psicosociales. En A. Salvia (Ed.), Tiempo de balance: deudas sociales pendientes al final del Bicentenario. Necesidad de atender las demandas del desarrollo humano con mayor equidad e inclusión social (pp. 173-212). Ciudad Autónoma de Buenos Aires: Educa.

Sluzki, C. (1996). La red social: frontera de la práctica sistémica. Barcelona: Gedisa.

Thoits, P. (1983). Multiple identities and psychological well-being: a reformulation and test of the social isolation hipótesis. American Sociological review, 48, 174-187. https://doi.org/10.2307/2095103

Universidad Católica Argentina (2016). Encuesta de la Deuda Social. Observatorio de la Deuda Social Argentina. Boletin $n^{\circ}$ 7. Buenos Aires: UCA.

Vera Noriega, J., Sotelo Quiñones, T., \& Dominguez Guedeas, M. (2005). Bienestar subjetivo, enfrentamiento y redes de apoyo social en adultos mayores. Revista Intercontinental de Psicologia y Educación, 7(2), 57-78.

Villalba Quesada, C. (1993). Redes sociales: Un concepto con importantesimplicaciones en la intervención comunitaria, Intervención Psicosocial, 2(4), 69-85.

Vivaldi, F., \& Barra, E. (2012). Bienestar psicológico, apoyo social percibido y percepción de salud en adultos mayores. Terapia psicológica, 30(2), 23-29. https://doi.org/10.4067/S071848082012000200002

Walker, K. (1977). Social support networks and the crisis of bereavement. Social Science and Medecine, 11(1), 35-41. https://doi.org/10.1016/0037-7856(77)90143-3

Wrzus, C., Hänel, M., Wagner, J., \& Neyer, F. J. (2013). Social network changes and life events across the lifes pan: A meta-analysis. Psychological bulletin, 139(1), 53-80. https://doi.org/10.1037/a0028601

\section{Recibido 02-06-2018 | Aceptado 08-03-2019}

